OPEN ACCESS

Edited by:

Jie Li,

Harbin Institute of Technology, China

Reviewed by:

Sayan Chatterjee,

Guru Gobind Singh Indraprastha

University, India

Frederico Marianetti Soriani,

Federal University of Minas Gerais,

Brazil

*Correspondence:

Jing Ye

yjholly@email.ncu.edu.cn

${ }^{+}$These authors have contributed equally to this work

Specialty section:

This article was submitted to

Molecular Diagnostics and

Therapeutics,

a section of the journal

Frontiers in Molecular Biosciences

Received: 18 December 2020 Accepted: 26 April 2021

Published: 28 May 2021

Citation:

Yu J, Kang X, Xiong Y, Luo Q, Dai D and Ye J (2021) Gene Expression

Profiles of Circular RNAs and MicroRNAs in Chronic Rhinosinusitis With Nasal Polyps.

Front. Mol. Biosci. 8:643504. doi: 10.3389/fmolb.2021.643504

\section{Gene Expression Profiles of Circular RNAs and MicroRNAs in Chronic Rhinosinusitis With Nasal Polyps}

\author{
Jieqing Yu ${ }^{1,2 \dagger}$, Xue Kang ${ }^{1,3 \dagger}$, Yuanping Xiong ${ }^{1}$, Qing Luo ${ }^{1}$, Daofeng Dai ${ }^{1}$ and Jing Ye ${ }^{1,2 *}$ \\ ${ }^{1}$ Department of Otorhinolaryngology Head and Neck Surgery, The First Affiliated Hospital of Nanchang University, Nanchang, \\ China, ${ }^{2}$ Jiangxi Otorhinolaryngology Head and Neck Surgery Institute, Nanchang, China, ${ }^{3}$ Department of Otorhinolaryngology \\ Head and Neck Surgery, Jiangxi Provincial Children's Hospital, Nanchang, China
}

Introduction: Chronic rhinosinusitis (CRS) is often classified primarily on the basis of the absence or presence of nasal polyps (NPS), that is, as CRS with nasal polyps (CRSwNP) or CRS without nasal polyps (CRSsNP). Additionally, according to the percentage of eosinophils, CRSwNP can be further divided into eosinophilic CRSwNP (ECRSWNP) and non-ECRSwNP. CRSwNP is a significant public health problem with a considerable socioeconomic burden. Previous research reported that the pathophysiology of CRSWNP is a complex, multifactorial disease. There have been many studies on its etiology, but its pathogenesis remains unclear. Dysregulated expression of microRNAs (miRNAs) has been shown in psoriasis, rheumatoid arthritis, pulmonary fibrosis, and allergic asthma. Circular RNAs (circRNAs) are also involved in inflammatory diseases such as rheumatoid arthritis, septic acute kidney injury, myocardial ischemia/reperfusion injury, and sepsis-induced liver damage. The function of miRNAs in various diseases, including CRSWNP, is a research hotspot. In contrast, there have been no studies on circRNAs in CRSwNP. Overall, little is known about the functions of circRNAs and miRNAs in CRSwNP. This study aimed to investigate the expression of circRNAs and miRNAs in a CRSwNP group and a control group to determine whether these molecules are related to the occurrence and development of CRSwNP.

Methods: Nine nasal mucosa samples were collected, namely, three ECRSwNP samples, three non-ECRSwNP samples, and three control samples, for genomic microarray analysis of circRNA and microRNA expression. All of the tissue samples were from patients who were undergoing functional endoscopic sinus surgery in our department. Then we selected some differentially expressed miRNAs and circRNAs for qPCR verification. Meanwhile, GO enrichment analysis and KEGG pathway analysis were applied to predict the biological functions of aberrantly expressed circRNAs and miRNAs based on the GO and KEGG databases. Receiver operating characteristic $(\mathrm{ROC})$ curve analysis and principal component analysis (PCA) were performed to confirm these molecules are involved in the occurrence and development of CRSWNP.

Results: In total, 2,875 circRNAs showed significant differential expression in the CRSwNP group. Specifically, 1794 circRNAs were downregulated and 1,081 circRNAs were upregulated. In the CRSwNP group, the expression of 192 miRNAs was significantly 
downregulated, and none of the miRNAs were significantly upregulated. GO and KEGG analysis showed differential circRNAs and miRNAs were enriched in "amoebiasis," "salivary secretion," "pathways in cancer," and "endocytosis." Through qRT-PCR verification, the expression profiles of hsa-circ-0031593, hsa-circ-0031594, hsa-miR132-3p, hsa-miR-145-5p, hsa-miR-146a-5p, and hsa-miR-27b-3p were shown to have statistical differences. In addition, ROC curve analysis showed that the molecules with the two highest AUCs were hsa-circ-0031593 with AUC 0.8353 and hsa-miR-145-5p with AUC 0.8690. Through PCA with the six ncRNAs, the first principal component explained variance ratio was $98.87 \%$. The AUC of the six ncRNAs was 0.8657 .

Conclusion: In our study, the expression profiles of ECRSwNP and non-ECRSwNP had no statistical differences. The differentially expressed circRNAs and miRNAs between CRSwNP and control may play important roles in the pathogenesis of CRSwNP. Altered expression of hsa-circ-0031593 and hsa-miR-145-5p have the strongest evidence for involvement in the occurrence and development of CRSWNP because their AUCs are higher than the other molecules tested in this study.

Keywords: chronic rhinosinusitis with nasal polyps, circular RNA, micro RNA, microarray analysis, gene express profile

\section{INTRODUCTION}

Chronic rhinosinusitis with nasal polyps (CRSwNP) is a significant public health problem with a considerable socioeconomic burden. Chronic rhinosinusitis (CRS), which is characterized by persistent mucosa inflammation of the sinuses, is one of the most common chronic diseases, and its pathophysiology remains unclear (AI-Sayed et al., 2017).

According to whether nasal polyps exist or not, CRS is often classified as CRSwNP or CRS without nasal polyps (CRSsNP) (Cho et al., 2017). CRSwNP remains a challenging clinical problem due to its propensity for recurrence. According to the percentage of eosinophils, CRSwNP can be classified as eosinophilic CRSwNP (ECRSwNP), with an eosinophil count $\geq 10 \%$, and non-eosinophilic CRSwNP (non-ECRSwNP), with an eosinophil count $<10 \%$. There are differences between the two subtypes of CRSwNP (Cao et al., 2009). Compared with nonECRSwNP, ECRSwNP is characterized by more eosinophils infiltrating the nasal mucosa, and it has a worse prognosis and higher recurrence rate (Shi et al., 2013). ECRSwNP and nonECRSwNP have different clinical symptoms, recurrence rates, and responses to drugs and endoscopic surgery (Lou et al., 2015). ECRSwNP is a hard-to-treat subtype of CRS.

To discover the pathogenesis of and better treatment for CRSwNP, more research is needed to further explore the different molecular and cytological mechanisms of the subtypes that lead to the different clinical and pathophysiological characteristics between the two subtypes.

With advancements in genomic microarray technology, a revolutionary change has taken place in the field of genetic analysis, that makes it possible to quantify thousands of gene expressions simultaneously. Studies have shown that the human genome can be widely transcribed into a large amount of noncoding RNAs (ncRNAs) that are closely related to the initiation as well as progression of diseases (Beermann et al., 2016). Genomic microarray technology has been widely used in the field of biomedicine to explore the occurrence and development of human diseases, including CRSwNP, at the genetic level (Plager et al., 2010; Yao et al., 2019).

CircRNAs (circular RNAs) are a type of ncRNA with important functions that have tissue specificity and disease specificity (Xia et al., 2017). Unlike linear RNAs (containing $5^{\prime}$ and $3^{\prime}$ ends), circRNAs are closed continuous loops that are free from exonuclease-mediated degradation and are more stable than most linear RNAs (Jeck et al., 2013). It has been found that circRNAs, acting as miRNA sponges, are rich in miRNA binding sites and increase the expression of target genes by mitigating the inhibition of miRNAs on their target genes (Kulcheski et al., 2016). CircRNAs represent a class of naturally occurring endogenous ncRNAs that have recently been recognized as important regulators of gene expression networks (Oude Voshaar et al., 2019). In recent years, researchers have explored the expression profiles of circRNAs in different diseases. For example, one study showed that oxidized lowdensity lipoprotein accelerates the injury of endothelial cells via the circ-USP36/miR-98-5p/VCAM1 axis (Peng et al., 2021). Another study found that circRNA_09505 aggravates inflammation and joint damage in RA via the miR-6089/ AKT1/NF-kB axis (Yang et al., 2020).

MiRNAs (microRNAs) are another group of ncRNAs that are involved in many pathologic and physiological processes, such as proliferation, differentiation, and tumorigenesis (Zhang X.-H. et al., 2012; Ferreira et al., 2018; Martínez-Rivera et al., 2018). Research has shown that the expression of miR-125 b is increased in ECRSwNP, which may lead to mucosal eosinophilia (Zhang Y.N. et al., 2012). In addition, miR-1 can regulate the transport of eosinophils in CRS. Overexpression of miR-1 inhibits the increase of airway eosinophils and inhibits the binding of eosinophils and 
endothelial cells induced by IL-13 (Korde et al., 2020). The interaction between circRNAs and miRNAs plays an important role in inflammation and immune responses. In psoriasis, circRNA-0061012 enhances GAB1 expression through spongy miR-194-5p, thereby promoting the proliferation, migration, and invasion of keratinocytes induced by IL-22 (He et al., 2021). CircRNA-WBSCR17 aggravates the inflammatory response of human renal tubular epithelial cells induced by high glucose by targeting the miR-185-5p/SOX6 axis (Li et al., 2020). In osteoarthritis, circRNA-9119 blocks the miR$26 a / P T E N$ axis to protect IL-1 $\beta$-treated chondrocytes from apoptosis (Chen et al., 2020). These reports show that circRNAs and miRNAs are related to inflammation.

To date, dysregulated expression of miRNAs has been shown in psoriasis, rheumatoid arthritis, pulmonary fibrosis, and allergic asthma. CircRNA is also involved in inflammatory diseases, but there is no research of circRNA in CRSwNP (Zhang Y.-N. et al., 2012). In our study, we aimed to compare the microarray expression profiles of miRNAs and circRNAs in nasal polyps of CRSwNP and normal nasal mucosa from control subjects. However, the results of RNA-seq genomic microarray analysis of ECRSwNP and non-ECRSwNP had no statistical differences, so, we combined ECRSwNP and non-ECRSwNP into a single group denoted as CRSwNP. Then, we validated the abnormally expressed circRNAs and miRNAs by qRT-PCR (quantitative real time polymerase chain reaction). In particular, we explored the potentially biological functions and involved signaling pathways of these ncRNAs by using the GO and KEGG databases. We concluded that the differentially expressed circRNAs and miRNAs may play important roles in the pathogenesis of CRSwNP. Based on ROC (receiver operating characteristic) curve analysis and principal component analysis (PCA), the altered expressions of hsa-circ-0031593, and hsa-miR-145-5p have the most evidence supporting their involvement in the occurrence and development of CRSwNP.

\section{MATERIALS AND METHODS}

\section{Subjects and Samples}

Nasal polyp specimens were collected from CRSwNP patients undergoing functional endoscopic sinus surgery. The middle turbinate mucosae of the control group were obtained from patients undergoing optic nerve decompression and nasal bone fracture surgery. Controls with nasal inflammation or upper respiratory tract infection were excluded. Subjects using corticosteroids or other immunemodulating drugs within 1 month, and subjects with antrochoanal polyps or fungal sinusitis were excluded. All of the participants were enrolled from the Department of Otorhinolaryngology Head and Neck Surgery, The First Affiliated Hospital of Nanchang University, Nanchang, China in 2017 (detailed information is shown in Supplementary Table S1). The study was approved by the Medical Research Ethics Committee of The First Affiliated Hospital of Nanchang University (2017080).

\section{Study Process}

The quantity of eosinophils in each specimen was observed by hematoxylin-eosin staining in three random microscopic high power fields (HPFs, $\times 400$ magnification). CRSwNP patients were classified according to the percentage of eosinophils in nasal polys. Nasal polyps from three ECRSwNP, three non-ECRSwNP, and three control individuals were collected for total RNA extraction and microarray analysis. We found that ECRSwNP and non-ECRSwNP tissues shared similar expression patterns of circRNAs and miRNAs. Therefore, we combined ECRSwNP and non-ECRSwNP into a single group denoted as CRSwNP. Then, the aberrant circRNAs and miRNAs with fold change $> \pm 2.5$ and $p<0.05$ were validated in an independent cohort (control group, $n=5$; CRSwNP, $n=5$ ) by qRT-PCR. Next, we expanded the sample size (control group, $n=25$; CRSwNP group, $n=29$ ) to conduct further research on the quantitative expression of the selected ncRNAs in the third cohort.

\section{Hematoxylin-Eosin Staining for Eosinophils}

The quantity of eosinophils was analyzed by hematoxylin-eosin staining. Specimens of nasal polyps were fixed in $10 \%$ formaldehyde and placed in low to high concentration alcohol to remove water from the tissues. Then the specimens were embedded in paraffin wax, sliced by a microtome into sections no more than $0.5 \mu \mathrm{m}$ thick, and deparaffinized to yield tissue sections. After rehydration, the tissue sections were stained with hematoxylin for $10 \mathrm{~min}$ and eosin for $3 \mathrm{~min}$. In order to classify CRSwNP into ECRSwNP and non-ECRSwNP, we calculated the percentage of eosinophils in all of the inflammatory cells through five random high-power fields. Specimens with eosinophils $\geq 10 \%$ were defined as ECRSwNP, and those with eosinophils $<10 \%$ as non-ECRSwNP (AI-Sayed et al., 2017).

\section{CircRNA Microarray Analysis}

The total RNAs were extracted from the subjects for microarray analysis. The purity and concentration of RNA were determined by the OD260/280 readings of a spectrophotometer (NanoDrop ND-1000). The integrity of RNAs was detected by standard denaturing agarose gel electrophoresis (Bioanalyzer 2100, Agilent Technologies, United States). The results are shown in Supplementary Figure S1. The digestion, amplification, and labeling of RNAs were performed based on the protocol provided by the manufacturer. The labeled RNAs were hybridized onto the microarray (Agilent-084217) after purification. The circRNA array data were analyzed by GeneSpring software V13.0 (Agilent). In order to select the differential expression of circRNAs, we used the threshold $\geq$ \pm 2.5 fold change and $p<0.05$.

\section{MiRNA Microarray Analysis}

MiRNA expression profile microarrays of these specimens were performed by CapitalBio. Procedures are described in detail on the CapitalBio website (http://www.capitalbio.com). Briefly, the procedure included total RNA extraction, quality control, miRNA isolation, FlashTag biotin labeling of miRNAs, hybridization to an Affymetrix GeneChip microarray (Affymetrix miRNA 4.0), and microarray washing, staining, 


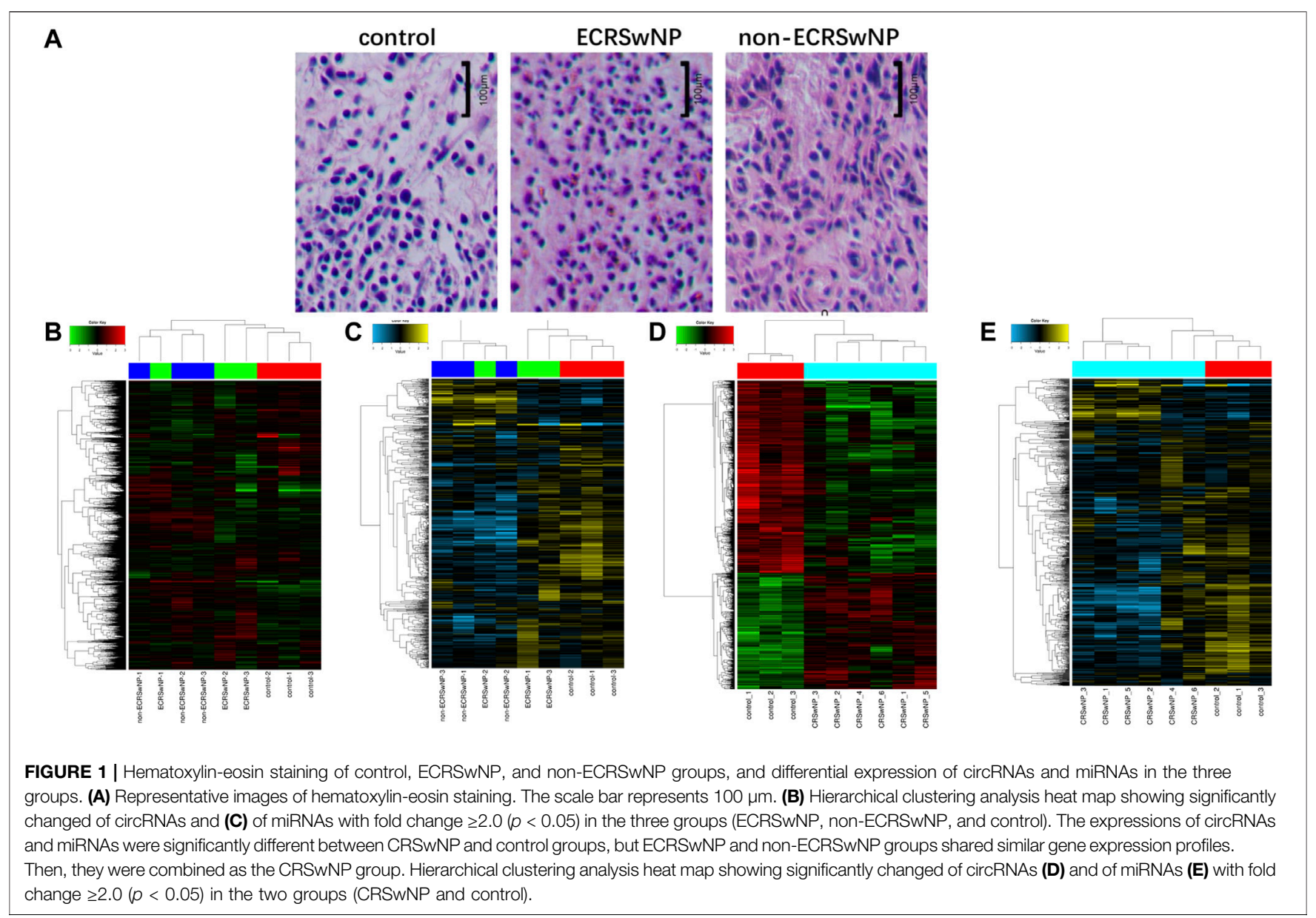

and scanning. If the miRNAs expression changed by at least \pm 2.5 fold $(p<0.05)$, this was considered a significant difference.

\section{Correlation and Co-Expression Analysis}

CircRNAs bind with miRNAs competitively, which inhibits the negative regulation of miRNAs on target genes and leads to the increase of the functional activity and expression of target genes (Fokkens et al., 2012). We constructed co-expression networks to predict the target genes of circRNAs and miRNAs of CRSwNP. The co-expression analysis was based on miRanda-3.3 software, with entropy values below 20. The top 40 circRNA-miRNA networks, $p<0.05$, were selected for analysis. In the network analysis, each point represents a gene, and two points connected by a line represent two closely related genes.

\section{Gene Ontology and Kyoto Encyclopedia of Genes and Genomes Pathway Analyses}

We identified functional categories that were significantly enriched relative to the background reference by GO enrichment analysis. The related pathways and gene interactions associated with the abnormal expression of circRNA and microRNAs were found based on the latest KEGG pathway enrichment database. The significant GO terms and pathways were determined by Fisher's exact test, and the false discovery rate was utilized to correct the $p$-values.

\section{Quantitative Real-Time Polymerase Chain Reaction for Validation of circRNAs and miRNAs}

The significant differential expression of circRNAs and miRNAs was quantified by qRT-PCR. Total RNA was isolated from nasal polyps with TRIzol reagent. The cDNAs were synthesized by reverse transcription with a PrimeScript RT reagent kit with random primers. Then, qRT-PCR was conducted by SYBR Premix Ex Taq II (Tli RNaseH Plus; TaKaRa). Primers for selected ncRNAs and house-keeping genes were synthesized by Sangon Biotech (Shanghai, China). The primers used are shown in Supplementary Tables S2, S3.

\section{Statistical Analysis}

SPSS 22.0 was used in this study. The Mann-Whitney U-test was used to calculate the differences of the expression of circRNAs and miRNAs between groups, and $p<0.05$ was considered to be statistically significant. The functional values of the selected circRNAs and miRNAs for CRSwNP were evaluated by conducting ROC curve analysis and PCA. 


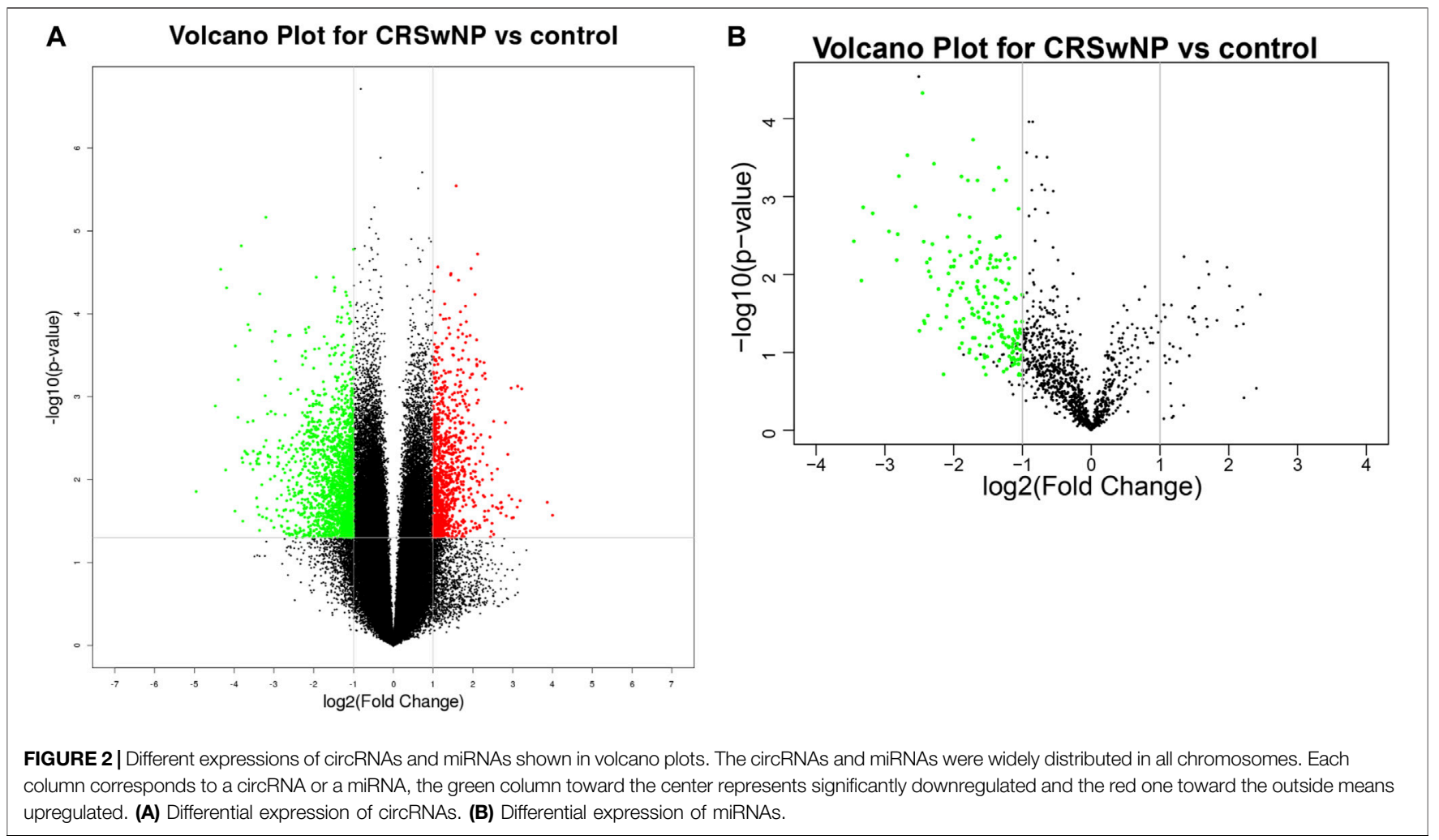

\section{RESULTS}

\section{ECRSwNP and Non-ECRSwNP Patients Shared Similar Gene Expression Profiles}

In this study, we used hematoxylin-eosin staining for eosinophil counts (Figure 1A). Hierarchical clustering analysis was used for evaluating gene expression differences among groups. As shown in Figures 1B,C, the ECRSwNP and non-ECRSwNP groups shared similar gene expression profiles, so we could not distinguish ECRSwNP from non-ECRSwNP by hierarchical clustering analysis. Then, we combined the ECRSwNP group and non-ECRSwNP group into the CRSwNP group. The results showed that the expression profiles of circRNAs (Figure 1D) and miRNAs (Figure 1E) were significantly different between the CRSwNP and control groups.

\section{Differential Expression of circRNAs and miRNAs in CRSwNP}

Volcano plots were used to assess the locations of circRNAs (Figure 2A) and miRNAs (Figure 2B). These ncRNAs were widely distributed in all of the chromosomes. Circos plots and scatter plots were also applied to analyze the gene expression differences between the CRSwNP and control groups (Supplementary Figures S2, S3). CircRNAs and miRNAs downregulated or upregulated with fold change $\geq \pm 2.5(p<0.05)$ in both the ECRSwNP group and nonECRSwNP group were considered to have significant differential expression and were selected for further research. A total of 2,875 circRNAs showed significant differential expression in the CRSwNP group, including 1,794 downregulated circRNAs and 1,081 upregulated circRNAs. Additionally, 192 miRNAs were significantly downregulated and no miRNAs were significantly upregulated in the CRSwNP group.

\section{Co-Expression Network in CRSwNP}

From gene co-expression network analysis, we found that there was a difference in the co-expression of circRNAs and miRNAs between the CRSwNP group and control group, which revealed the underlying molecular mechanism of the pathogenesis of CRSwNP. We selected 40 circRNAs differentially expressed between the CRSwNP and control groups. The top 40 circRNAs-miRNAs networks are shown in Figure 3. Hsa-circ-0031593, hsa-circ-0031594, and hsamiR-27b-3p are present in Figure 3. One circRNA can be associated with multiple miRNAs, and one miRNA can be related to multiple circRNAs, resulting in complex functional connections. In Figure 3, the darker and larger nodes indicate the higher fold change of circRNAs, purple indicates upregulation, and blue indicates downregulation. There were many regulatory relationships between the circRNAs and miRNAs in the networks, which further indicated that the networks of regulatory relationships were ubiquitous. 


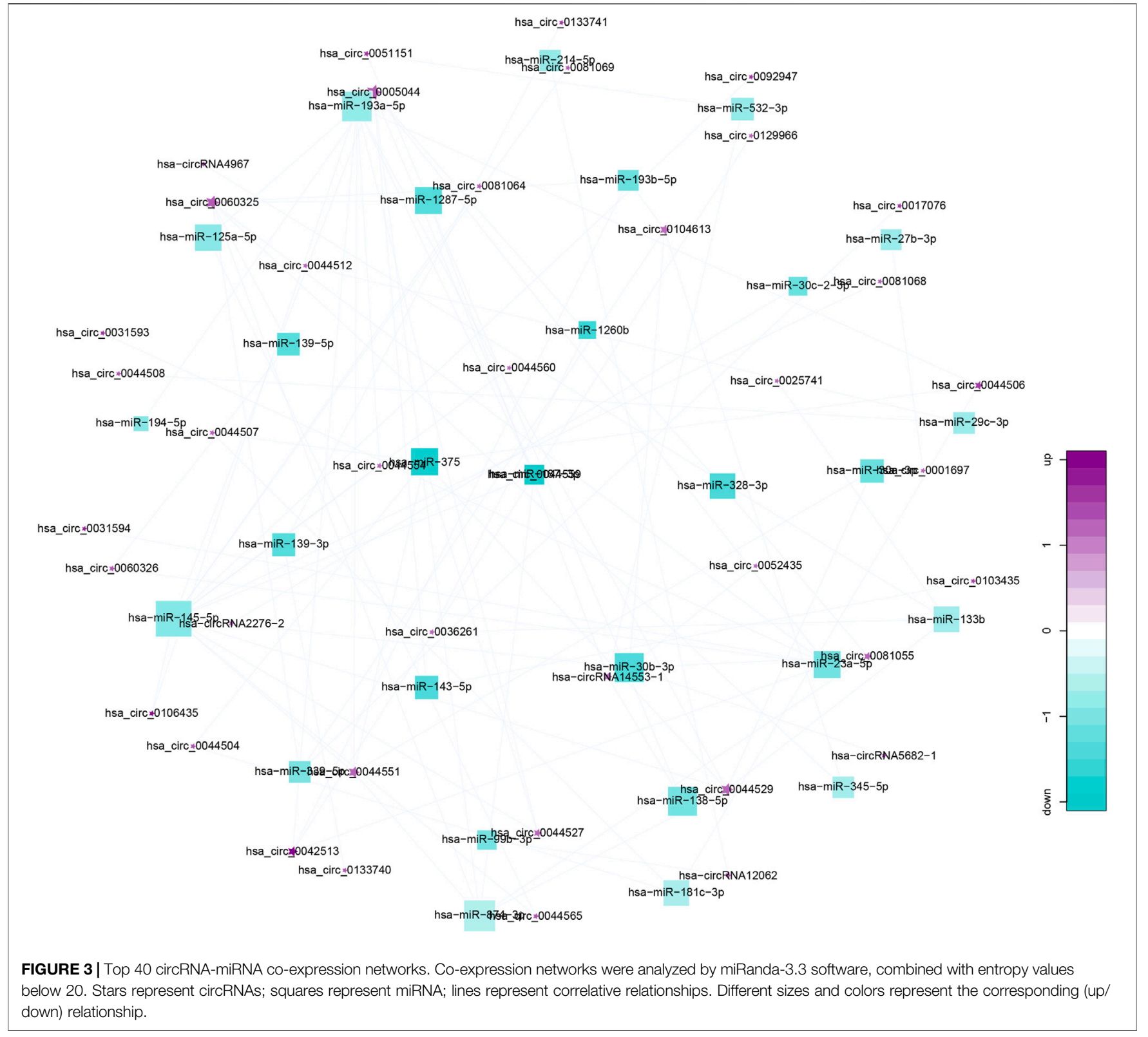

\section{Gene Ontology and Kyoto Encyclopedia of Genes and Genomes Pathway Analyses}

We predicted the biological functions of aberrantly expressed circRNAs and miRNAs through GO enrichment analysis and KEGG pathway analysis. The enriched GO terms $(p<0.05)$ could reflect the function of aberrantly expressed circRNAs and miRNAs. Each plot represents a specific biological function, and a larger size means a larger gene number. For circRNAs, compared with the normal control subjects, the significantly over-presented terms were mainly involved in cell adhesion, membrane, and receptor activity. In contrast, miRNAs were mainly involved in cellular component organization, intracellular parts, protein binding, and so on. The details of the top 10 significantly enriched GO terms of circRNAs are presented in Figure 4. According to GO analysis of circRNAs in the biological process (BP) category, the differentially expressed circRNAs were chiefly enriched in "single-organism process" and "single-organism cell process" (Figure 4). GO analysis related to cellular components (CC) showed that they were mainly enriched in "membrane" and "membrane part" (Figure 4). GO analysis related to molecular functions (MF) demonstrated that they were involved in "molecular transducer activity" and "receptor activity" (Figure 4). The GO analysis of miRNAs is shown in Figure 5. The biological process category was enriched in "positive regulation of biological process" and "anatomical structure development" (Figure 5). Cellular components (CC) were mostly enriched in "intracellular" and "intracellular part" (Figure 5). Molecular functions (MF) were involved in "binding" and "protein binding" (Figure 5). In 


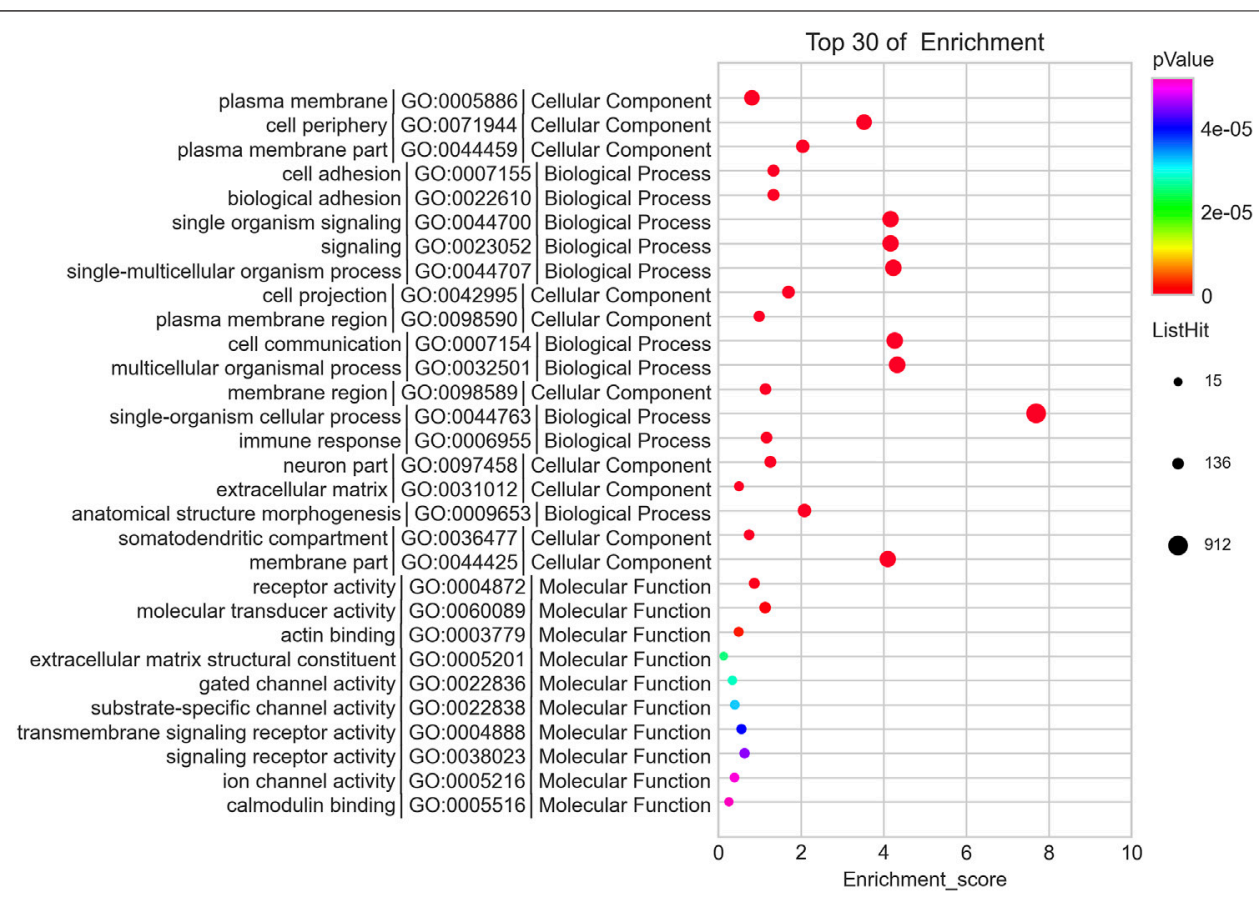

FIGURE 4 | Top 10 significant enriched GO terms analysis of circRNAs. Differentially expressed circRNAs were mainly involved in biological process, cellular component, and molecular function. Each plot represents a specific biological function, and the larger the size, the stronger the circRNAs expression. Compared with the normal subjects, the significantly over-presented circRNAs in the CRSwNP group were mainly involved in cell adhesion, membrane, and receptor activity.

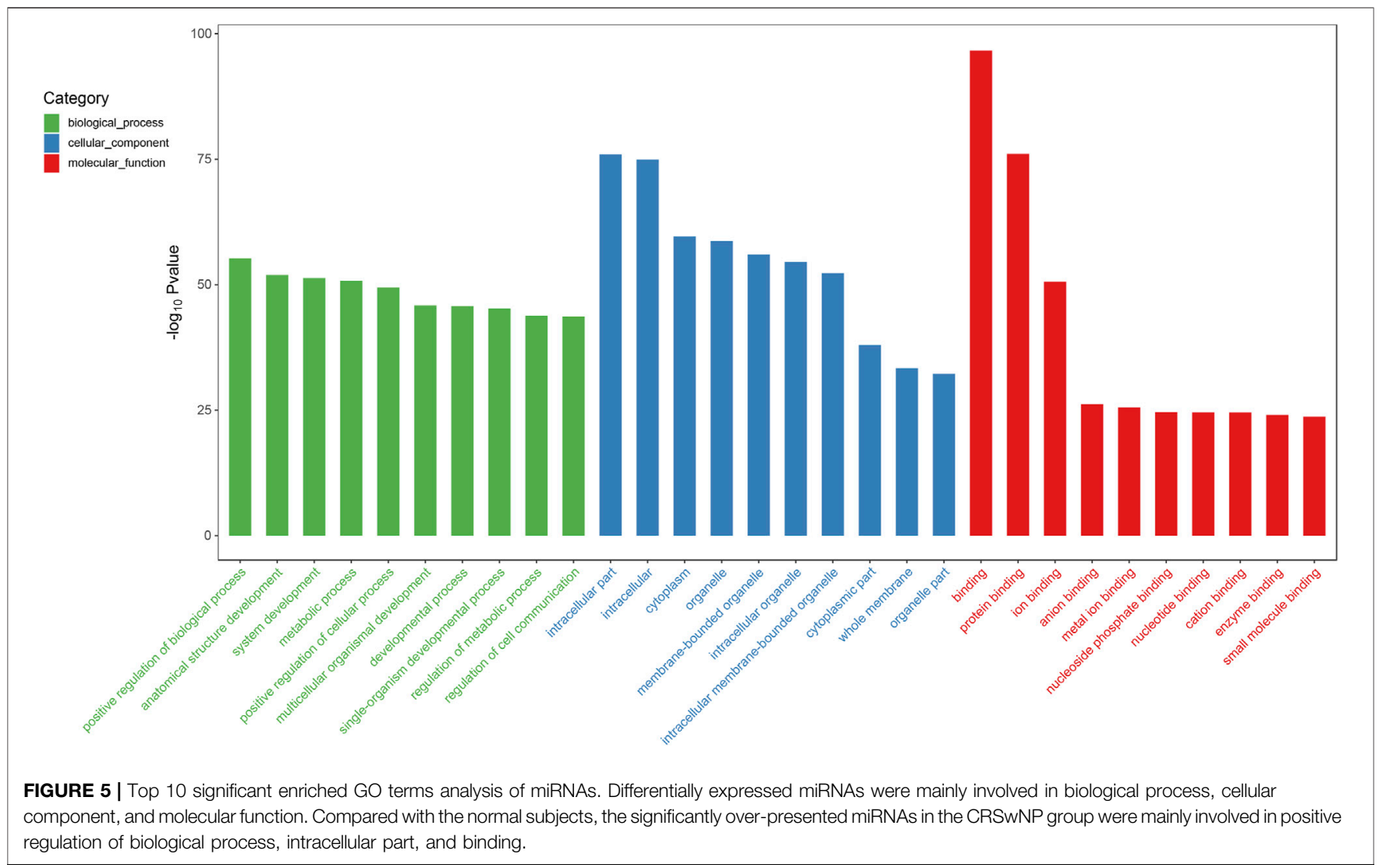




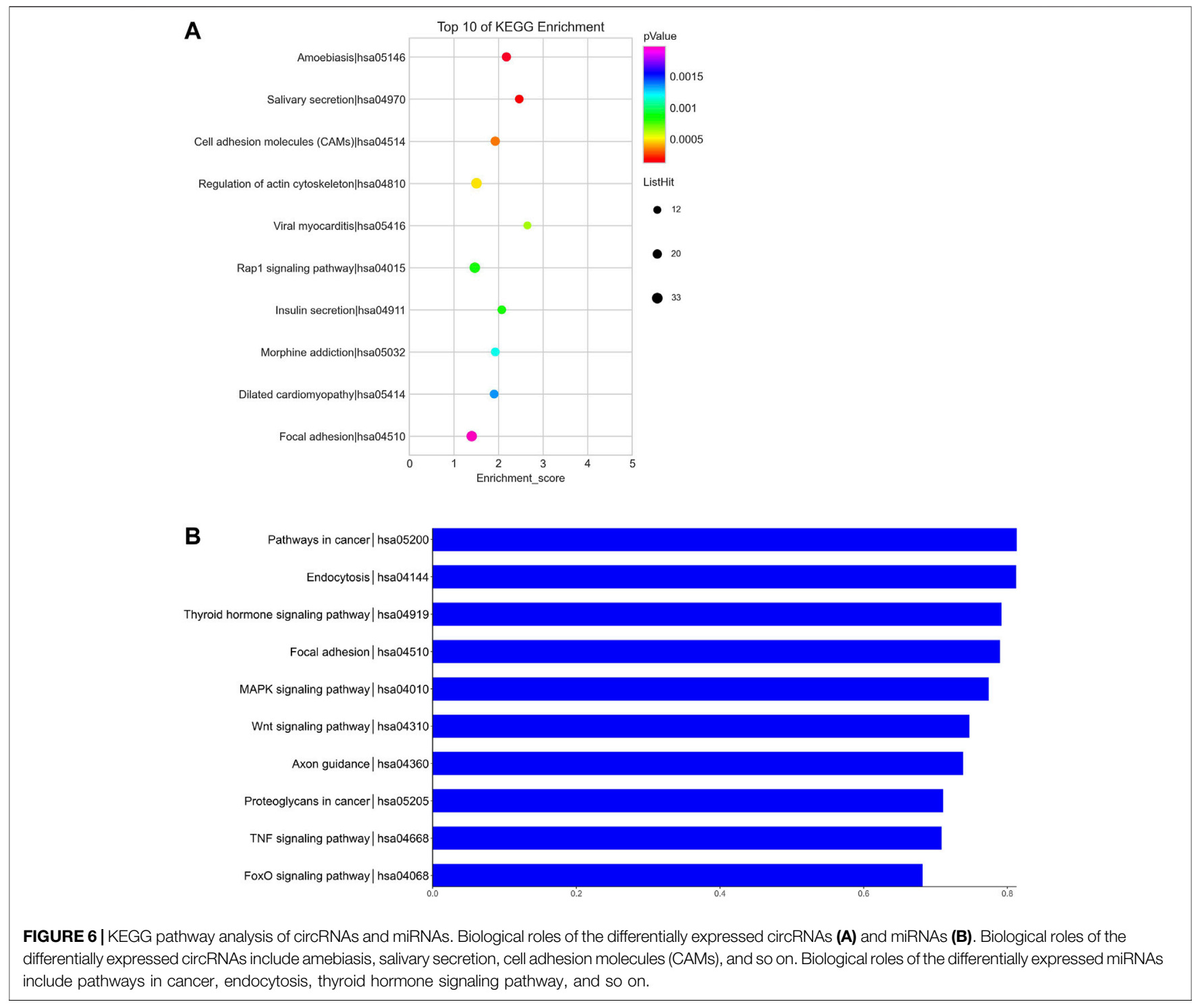

addition, from KEGG pathway analysis, we found that differentially expressed circRNAs were annotated to amebiasis, salivary secretion, and others (Figure 6A); and miRNAs were annotated to pathways in cancer, endocytosis, and so on (Figure 6B).

\section{Confirmation of the Expression of circRNAs and miRNAs Using RNA-Sequencing and Quantitative Real-Time Polymerase Chain Reaction}

The results of RNA-seq analysis showed that hsa-circ-0031593 and hsa-circ-0031594 were expressed to a significantly higher degree in the CRSwNP group than in the control group. Hsa-circ0109623, hsa-circ-0000736, hsa-miR-125a-5p, hsa-miR-132-3p, hsa-miR-145-5p, hsa-miR-146a-5p, and hsa-miR-27b-3p were to a significantly lower degree in the CRSwNP group than in the control group (Figure 7A). To confirm the reliability of the microarray results, circRNAs and miRNAs downregulated and upregulated with fold change $\geq \pm 2.5(p<0.05)$ in five CRSwNP subjects and five control subjects were selected for qRT-PCR in order to analyze the expression levels of these ncRNAs. The results showed that hsa-circ-0031593 and hsa-circ-0031594 were expressed significantly more in the CRSwNP group than in the control group. Hsa-miR-132-3p, hsa-miR-145-5p, hsa-miR$146 a-5 p$, and hsa-miR-27b-3p were expressed significantly less in the CRSwNP group than in the control group. There were no statistical differences among the expressions of hsa-circ-0109623, hsa-circ-0000736, or hsa-miR-125a-5p (Figure 7B).

After that, more samples (29 CRSwNP subjects and 25 control subjects) were used for further research of the quantitative expressions of these circRNAs and miRNAs. The detailed expression levels of these ncRNAs are shown in Figure 8. The results in Figure 8 are in accordance with the results in Figure 7B. The expressions of hsa-circ-0031593 and hsa-circ-0031594 in the CRSwNP group were significantly higher than those in the 

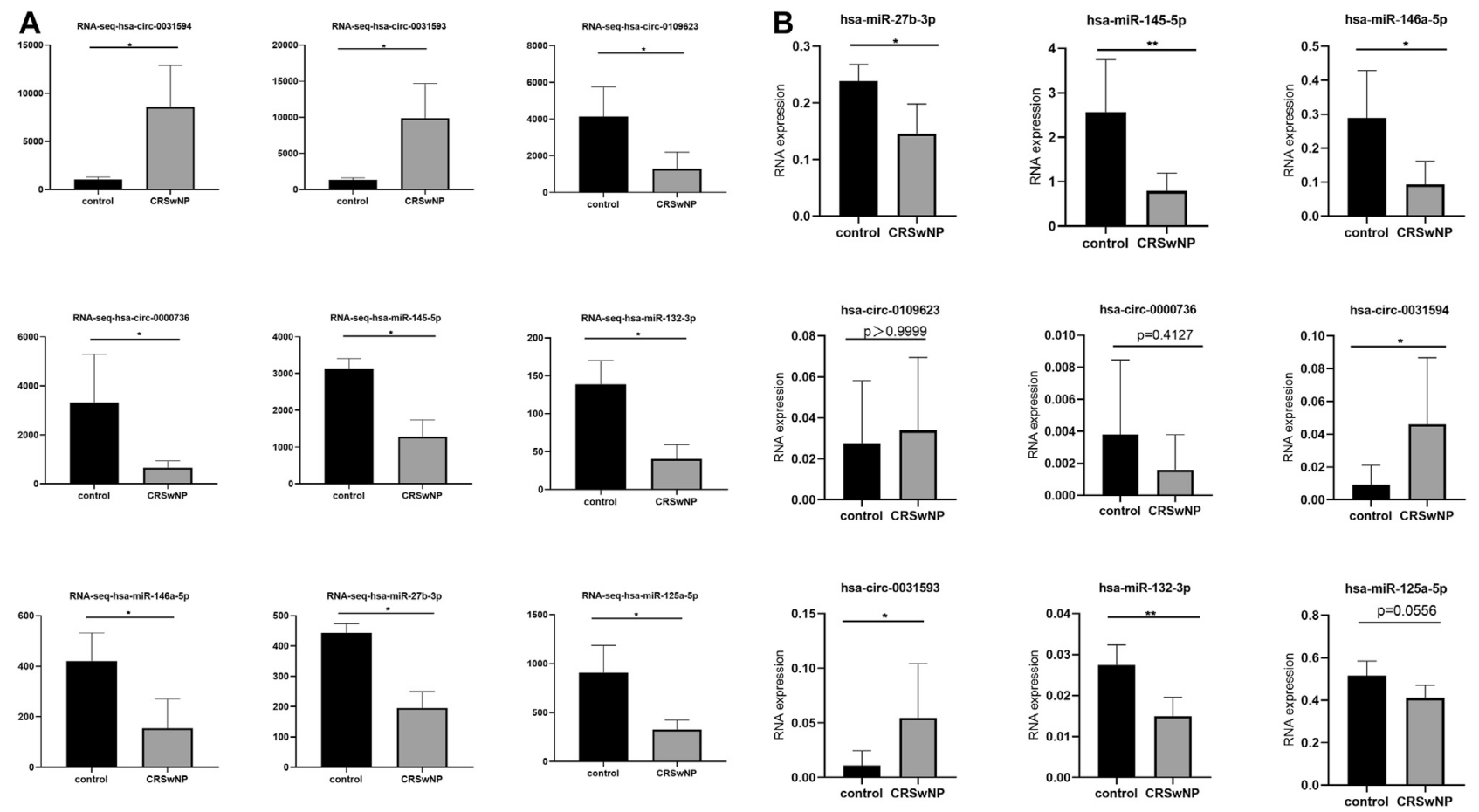

FIGURE 7 | Confirmation of the expression of circRNAs and miRNAs using RNA-sequencing and quantitative real-time polymerase chain reaction (qRT-PCR). CircRNAs and miRNAs were validated by RNA-sequencing (CRSwNP( $n=6)$; control subjects $(n=3)(\mathbf{A})$; and qRT-PCR in an independent cohort of five CRSwNP subjects and five control subjects (B). Each sample was detected in triplicate. GAPDH was used as the reference gene for circRNAs, and hsa-miR-16 was used as the reference gene for miRNAs. The relative expression levels of hsa-circ-0031593, hsa-circ-0031594, hsa-circ-0109623, hsa-circ-0000736, hsa-miR-132-3p, hsamiR-145-5p, hsa-miR-146a-5p, hsa-miR-125a-5p, and hsa-miR-27b-3p by qRT-PCR. ${ }^{\star} p<0.05,{ }^{\star \star} p<0.01,{ }^{\star \star \star} p<0.001,{ }^{\star \star \star \star} p<0.0001$.

control group. The expressions of hsa-miR-132-3p, hsa-miR-145$5 p$, hsa-miR-146a-5p, and hsa-miR-27b-3p in the CRSwNP group were significantly lower than those in the control group. There were no statistical differences among the expressions of hsa-circ-0109623, hsa-circ-0000736, or hsa-miR-125a-5p.

\section{ROC Curve Analysis and PCA of Selected circRNAs and miRNAs}

ROC curve analysis was carried out to estimate the functional values of the selected circRNAs and miRNAs in the occurrence and development of CRSwNP (Figure 9). The sensitivity and specificity for the values of CRSwNP are shown in Table 1. The PCA method was used in this research. The data of PCA with combinations of hsa-circ-0031593, hsa-circ-0031594, hsa-miR132-3p, hsa-miR-145-5p, hsa-miR-146a-5p, and hsa-miR-27b-3p are displayed in Table 2. The first principal component explained variance ratio was $98.87 \%$. We used the first principal component 1 of these six ncRNAs to carry out ROC curve analysis, and the AUC was 0.8657, indicating a good significance for the pathogenesis of CRSwNP. The AUCs of hsa-circ-0031593 and hsa-miR-145-5p were 0.8353 [(0.7291-0.9415), $p<0.0001]$ and 0.8690 [(0.76-0.978), $p<0.0001]$. Hsa-circ-0031593 and hsamiR-145-5p had the strongest evidence supporting their involvement in the occurrence and development of CRSwNP since they had higher AUCs than others and had $p$ values $<0.05$.

\section{DISCUSSION}

CRSwNP is a significant public health problem with a considerable socioeconomic burden. Previous studies have reported that CRSwNP is a complex, multifactorial disease. There have been many studies on its etiology, but its pathogenesis remains unclear. Dysregulated expression of miRNAs has been shown in psoriasis, rheumatoid arthritis, pulmonary fibrosis, and allergic asthma. CircRNA is also involved in inflammatory diseases, but there has been no research on the role of circRNA in CRSwNP. Although numerous researchers have attempted to clarify the pathogenesis of CRSwNP, the detailed mechanisms remain unclear. Overall, little is known on the role of ncRNAs in the pathogenesis of CRSwNP. Further understanding of the genetic level of pathogenesis is essential for developing new techniques for effective prevention and therapy to improve prognosis. Researchers have found that ncRNAs, such as circRNAs and miRNAs, play essential roles in the occurrence and development of many diseases, which is contrary to the traditional view that genes are mainly regulated by protein coding (Wu et al., 2019; Yang et al., 2019). To explore the functions of circRNAs and miRNAs in CRSwNP, we performed gene microarray analysis of circRNAs and miRNAs in a CRSwNP group and a control group. Functional enrichment analysis and prediction of differentially expressed genes were carried out by using public databases. In addition, we performed qRT-PCR to validate the reliability of RNA-seq analysis, and we confirmed that hsa-circ-0031593 and 


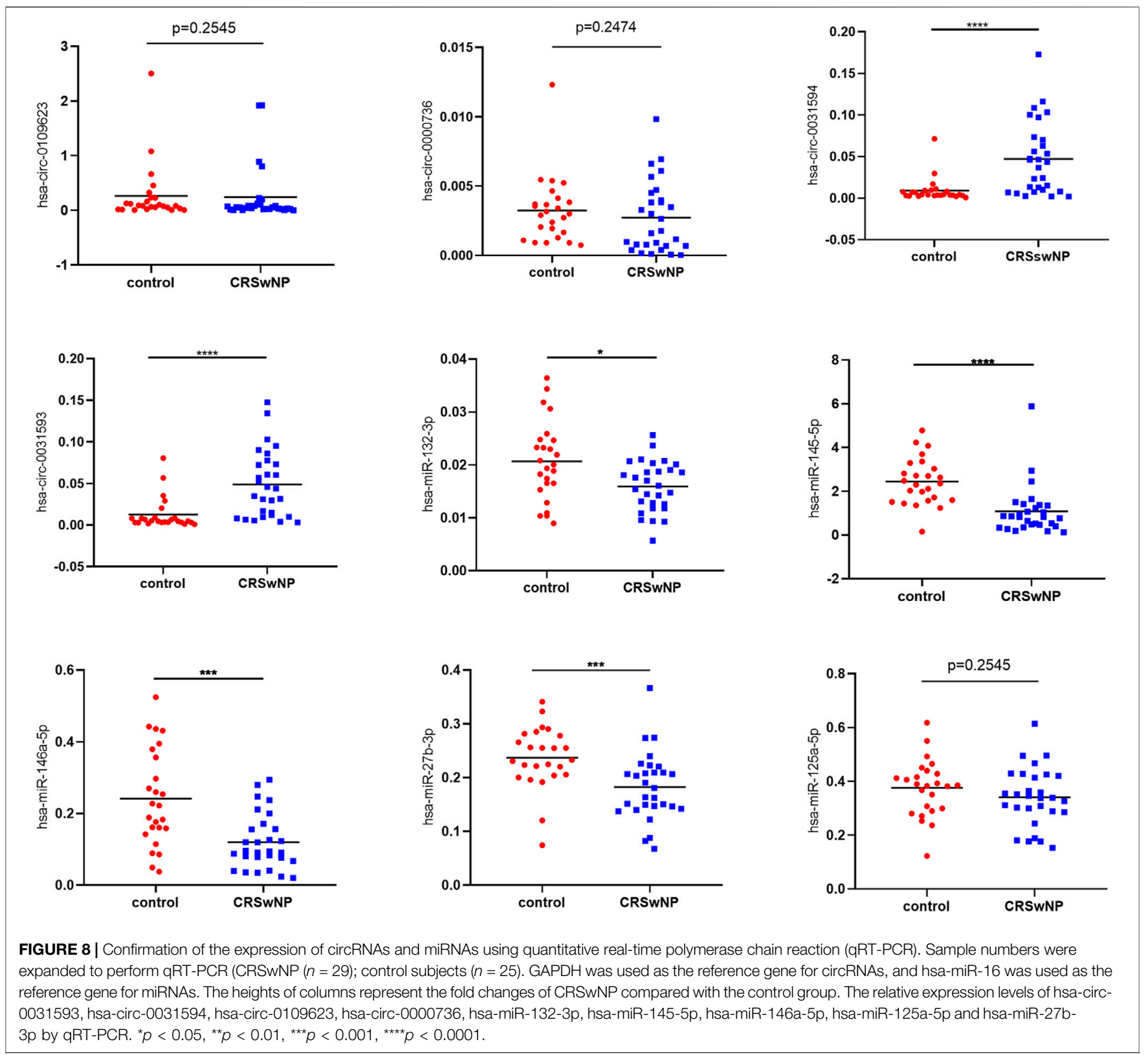

hsa-miR-145-5p had the strongest evidence supporting their involvement in the occurrence and development of CRSwNP by ROC curve analysis and PCA. In conclusion, our findings revealed a network potentially involved in CRSwNP pathogenesis, in which circRNAs and microRNAs play significant roles.

ECRSwNP differs greatly from non-ECRSwNP in many aspects (Shah et al., 2016; Lou et al., 2018), such as pathogenesis, development, prognosis, and CT scan images. Our original intention was to explore the functions of miRNA and circRNA in different CRSwNP subtypes, in order to help postoperative treatments like determining the eosinophil count. In addition, some researchers have found that ECRSwNP is difficult to treat and has a high recurrence rate, leading to poor clinical outcomes. However, in our study, the expressions of circRNA and miRNA between ECRSwNP and non-ECRSwNP had no statistical differences. It is possible that individual differences of the samples or the regulation of the next biological process has changed, resulting in different types of polyps. The development process of CRSwNP is complex and diverse (Schleimer, 2017). Meanwhile, the classification between ECRSwNP and non-ECRSwNP is limited. First, there is no unified view on the determination of ECRSwNP throughout the world. Second, the count of eosinophils is objective. Therefore, we combined ECRSwNP and non-ECRSwNP into the CRSwNP group.

After analyzing the different expressions of circRNAs and miRNAs between the CRSwNP and control groups, we found that 1794 circRNAs were significantly downregulated and 1,081 were significantly upregulated in the CRSwNP group. Additionally, 192 miRNAs showed significant downregulation in the CRSwNP 


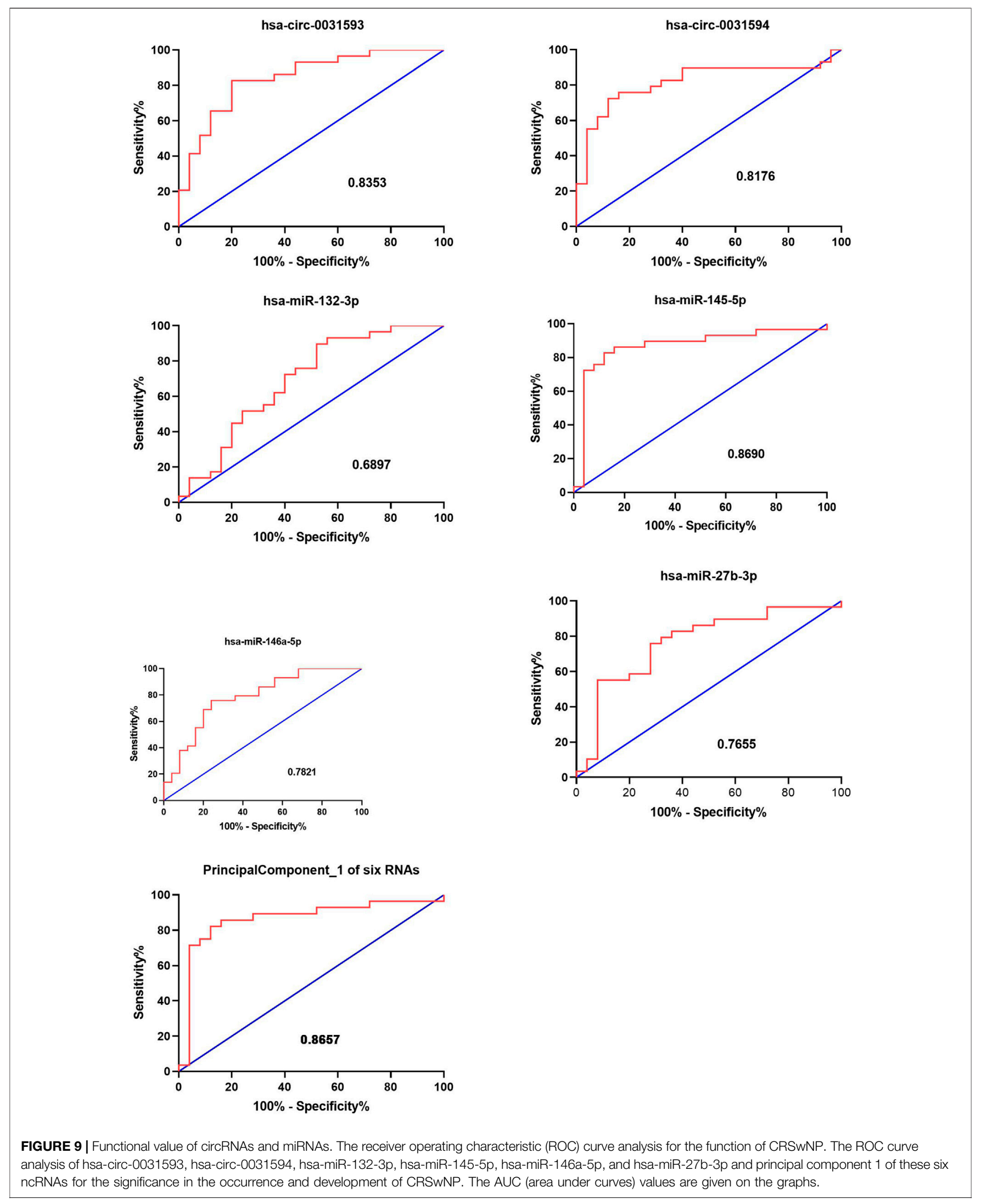


TABLE 1 | Validation of the selected circRNAs and miRNAS by quantitative real-time polymerase chain reaction and the data of ROC curve analysis.

\begin{tabular}{|c|c|c|c|c|c|}
\hline & AUC & $95 \% \mathrm{Cl}$ & $p$ value & Sensitivity & Specificity \\
\hline Hsa-circ-0031593 & 0.8353 & $0.7291-0.9415$ & $<0.0001$ & 0.8235 & 0.80 \\
\hline Hsa-circ-0031594 & 0.8176 & $0.7047-0.9306$ & $<0.0001$ & 0.7059 & 0.88 \\
\hline Hsa-miR-132-3p & 0.6897 & $0.5438-0.8355$ & 0.0171 & 0.8966 & 0.48 \\
\hline Hsa-miR-145-5p & 0.8690 & 0.76-0.978 & $<0.0001$ & 0.8276 & 0.88 \\
\hline Hsa-miR-146a-5p & 0.7821 & $0.6579-0.9063$ & 0.0004 & 0.7586 & 0.76 \\
\hline Hsa-miR-27b-3p & 0.7655 & $0.6332-0.8979$ & 0.0008 & 0.7586 & 0.72 \\
\hline Principal component 1 of six RNAs & 0.8657 & $0.7547-0.9768$ & $<0.0001$ & 0.8214 & 0.88 \\
\hline
\end{tabular}

AUC, area under curves.

group and none showed upregulation. The different expressions of circRNAs and miRNAs between these two groups may be involved in the pathogenesis of CRSwNP.

Studies have shown that one of the important functions of circRNAs is to act as "miRNA sponges" and competitively bind miRNAs to regulate post-transcriptional activity (Ergun and Oztuzcu, 2015). Co-expression networks have been constructed to obtain the relationship between circRNAs and miRNAs. Figure 3 shows that a single circRNA is associated with multiple miRNAs, and a single miRNA is associated with multiple circRNAs. Little is known about the relationship between circRNAs and miRNAs in CRSwNP. In fact, the present study is the first to detect circRNA in CRSwNP.

Without functional analysis, the huge quantity of data on gene expression is unintelligible. Hence, functional enrichment analysis and prediction of differentially expressed genes were carried out using public databases, such as GO enrichment analysis and KEGG pathway analysis, and we found that differential expressions of circRNAs between the CRNwNP and control groups were related to "amoebiasis," "salivary secretion," "cell adhesion molecules (CAMs)," "cAMP signaling pathway," "focal adhesion", "adherens junction," "TNF signaling pathway," and others. The differential expressions of miRNAs between the CRSwNP and control groups were enriched in "pathways in cancer," "endocytosis," "thyroid hormone signaling pathway," "salivary secretion," "regulation of actin cytoskeleton," "insulin secretion," and so on. Based on the functional analysis, the most significantly enriched pathway was CAMs, which was consistent with previous research (Milonski et al., 2015; Xu et al., 2017). The pathological characteristics of CRSwNP include inflammatory cells migrating to and infiltrating the nasal mucosa. During different stages of the progression of CRSwNP, the expressions of CAMs are various, which stimulate eosinophil and mast cell aggregation and contribute to Th2 skewing (Oyer et al., 2013). Compared to normal nasal mucosa, CRSwNP was shown to be more sensitive to IL-32 through lipopolysaccharides acting at the cAMP signaling pathway (Cho et al., 2016). Further, a study found that thromboxane $\mathrm{A} 2$ is involved in platelet aggregation and tissue inflammation in CRS, and cAMP regulates the expression of the thromboxane-prostanoid receptor and $\mathrm{cxcl} 1 / 8$, which participates in the pathogenesis of CRS (Elion et al., 2018). TNF, a complex and important inflammatory factor, induces local production of IgA and stimulates eosinophils, and it plays an important role in the pathogenesis of CRSwNP (Kato et al., 2008; Cho et al., 2015; Shimizu et al., 2016). The integrity of the airway epithelium is a prerequisite for its good barrier function, which depends on the intercellular junctions, including tight junctions and adhesion junctions (Suzuki et al., 2016; Jiao et al., 2018). Studies have shown that the breakdown of tight junctions and adhesion junctions of CRS and the decrease of protein component expression are major factors leading to the occurrence of CRS (Kim et al., 2018; Tian et al., 2018). Studies on the key genes and pathways in CRSwNP showed that salivary secretion was the most significantly enriched pathway for downregulated genes, which was consistent with our findings (Yao et al., 2019). Above all, our findings confirmed the validity of previous research and showed high reliability. Little is known about the regulation of the actin cytoskeleton and insulin secretion in CRSwNP. Studies have shown that the actin cytoskeleton is associated with several inflammatory diseases and is involved in leukocyte transendothelial migration (Schnoor, 2015; Ao et al., 2016; Lechuga and Ivanov, 2017). Besides, numerous studies have shown that the actin cytoskeleton plays an important role in regulating insulin secretion (Martínez-García et al., 2015; Sorrenson et al., 2016; Deyev et al., 2017). However, the functions of the actin cytoskeleton and insulin secretion in CRSwNP need to be further explored.

The heterogeneity of CRSwNP is gradually being recognized, prompting the discovery of novel biomarkers to describe specific endotypes and determine optimized treatment (Dennis et al., 2016; Kuhar et al., 2017). Recent studies on potential biomarkers in CRSwNP mainly focused on eosinophils, exhaled gas components, and inflammatory cells in nasal secretions, nasal tissues, and peripheral blood (Drake et al., 2016; Tsybikov et al., 2016; Asano et al., 2017; Chen et al., 2017). Yan indicated that miR$145-5 p$ negatively regulates the proliferation and chemokine secretion of NHEKs by targeting MLK3, and the downregulation of miR-145-5p contributes to skin inflammation in psoriasis lesions (Yan et al., 2019). Dihydroquercetin attenuates lipopolysaccharideinduced acute lung injury by modulating FOXO3-mediated NF- $\kappa \mathrm{B}$ signaling via miR-132-3p (Liu J.-H. et al., 2020). Human neutrophil elastase induces MUC5AC overexpression in chronic rhinosinusitis through miR-146a (Yan et al., 2020). Furthermore, miR-27b-3p, miR-181a-1-3p, and miR-326-5p are involved in the inhibition of macrophage activation in chronic liver injury (Li et al., 2017). Circ_0134111 knockdown relieves IL-1 $\beta$-induced apoptosis, inflammation, and extracellular matrix degradation in human chondrocytes through the circ_0134111-miR-515-5p-SOCS1 network (Wu et al., 2021). An inducible circular RNA circKcnt2 inhibits ILC3 activation to facilitate colitis resolution (Liu B. et al., 2020). These studies show that circRNA and miRNA play vital functions in the process of inflammation. In the present study, ROC curve analysis and PCA indicated that aberrantly expressed circRNAs and miRNAs may be related to biological dysfunction 
TABLE 2 | The data of principal component 1 by PCA with combinations of hsacirc-0031593, hsa-circ-0031594, hsa-miR-132-3p, hsa-miR-145-5p, hsamiR-146a-5p, and hsa-miR-27b-3p.

\begin{tabular}{|c|c|}
\hline Principal component 1 & Type of sample \\
\hline-0.207152043 & Control \\
\hline 2.500343003 & Control \\
\hline 0.987185596 & Control \\
\hline 1.304084045 & Control \\
\hline-0.361852501 & Control \\
\hline 0.755023033 & Control \\
\hline-1.570053914 & Control \\
\hline-0.481308455 & Control \\
\hline 0.906674823 & Control \\
\hline 0.30217685 & Control \\
\hline 2.353877347 & Control \\
\hline 1.965290304 & Control \\
\hline-0.28100719 & Control \\
\hline 1.564228751 & Control \\
\hline 3.054599405 & Control \\
\hline-0.126055013 & Control \\
\hline 0.567948512 & Control \\
\hline 1.087283413 & Control \\
\hline-0.009538338 & Control \\
\hline 0.62720042 & Control \\
\hline 0.255341755 & Control \\
\hline-0.162273621 & Control \\
\hline 0.38535951 & Control \\
\hline 1.632552725 & Control \\
\hline 0.966021961 & Control \\
\hline-1.33220639 & CRSWNP \\
\hline-0.361203299 & CRSWNP \\
\hline-0.386665708 & CRSWNP \\
\hline-1.597233545 & CRSWNP \\
\hline-1.396044339 & CRSWNP \\
\hline-1.205543467 & CRSwNP \\
\hline-1.376653623 & CRSWNP \\
\hline 4.157249255 & CRSWNP \\
\hline-0.902849991 & CRSWNP \\
\hline-0.860962834 & CRSWNP \\
\hline-0.22755494 & CRSwNP \\
\hline-1.464884451 & CRSwNP \\
\hline-0.876447159 & CRSwNP \\
\hline 0.712907312 & CRSWNP \\
\hline-0.962998826 & CRSwNP \\
\hline 1.21901289 & CRSWNP \\
\hline-0.685225616 & CRSWNP \\
\hline-1.19975492 & CRSWNP \\
\hline-1.560321 & CRSwNP \\
\hline-0.314747233 & CRSWNP \\
\hline-0.088432428 & CRSwNP \\
\hline-1.263311269 & CRSWNP \\
\hline-0.777328458 & CRSWNP \\
\hline-1.238227185 & CRSwNP \\
\hline-0.490926392 & CRSWNP \\
\hline-1.537602896 & CRSwNP \\
\hline-0.910574069 & CRSWNP \\
\hline-1.087419795 & CRSwNP \\
\hline
\end{tabular}

and play important roles in the pathogenesis of CRSwNP. Furthermore, hsa-circ-0031593 and hsa-miR-145-5p were more likely to correlate with the occurrence and development of CRSwNP.

There are still some limitations in our study. First, individual differences of the samples may have led to the lack of statistical differences between the ECRSwNP and non-ECRSwNP groups, although our findings were consistent with previous research (Cho et al., 2016; Suzuki et al., 2016; Xu et al., 2017; Kim et al., 2018; Yao et al., 2019). Second, all patients' data were from The First Affiliated Hospital of Nanchang University, and all patient were from Jiangxi Province. Although most of the Chinese population is Han, given that ethnic and regional variations may be involved in the development of CRSwNP, we will consider these variables in future studies. Third, our findings were only based on gene chip analysis, database comparison and prediction, and tissue experiment verification. Experiments in vivo and in vitro should be carried out to further explore the function of these aberrant genes in CRSwNP.

\section{CONCLUSION}

In our study, the expression profiles of ECRSwNP and non-ECRSwNP had no statistical differences. The differentially expressed circRNAs and miRNAs between the CRSwNP and control groups may play important roles in the pathogenesis of CRSwNP. Altered expression of hsa-circ-0031593 and hsa-miR-145-5p had the strongest evidence for involvement in the occurrence and development of CRSwNP.

\section{DATA AVAILABILITY STATEMENT}

The datasets presented in this study can be found in online repositories. The name of the repository and accession numbers can be found below: National Center for Biotechnology Information (NCBI) Gene Expression Omnibus (GEO), https:/www.ncbi.nlm.nih.gov/geo/, GSE169375 and GSE169376'

\section{ETHICS STATEMENT}

The studies involving human participants were reviewed and approved by the Department of Otorhinolaryngology and Head Neck Surgery, the First Affiliated Hospital of Nanchang University, Nanchang, China in 2017. The study was approved by the Medical Research Ethics Committee of the hospital (2017080). The patients/participants provided their written informed consent to participate in this study.

\section{AUTHOR CONTRIBUTIONS}

JYU, XK, and JYE conceived of or designed the work; JYU, XK, and JYE drafted the work; data acquisition was undertaken by YX and QL; data analysis was completed by DD; supervision or mentorship was done by JYU, YX, and XK. All authors contributed to the article and approved the submitted version.

\section{FUNDING}

This study was supported by National Natural Science Foundation of China (No. 81860182), Jiangxi Natural Science Foundation (No.20181BAB205036) and Jiangxi Natural Science Foundation (No. 20192BBGL70025). 


\section{ACKNOWLEDGMENTS}

We thank LetPub (www.letpub.com) for providing linguistic assistance during the preparation of this manuscript. Thank Yanqing Yu for her assistance in counting the tissue eosinophil number.

\section{REFERENCES}

Ai-Sayed, A. A., Agu, R. U., and Massoud, E. (2017). Models for the Study of Nasal and Sinus Physiology in Health and Disease: A Review of the Literature. Laryngoscope Invest. Otolaryngol. 2 (6), 398-409. doi:10.1002/lio2.117

Ao, M., Wu, L., Zhou, X., and Chen, Y. (2016). Methyl- $\beta$-Cyclodextrin Impairs the Monocyte-Adhering Ability of Endothelial Cells by Down-Regulating Adhesion Molecules and Caveolae and Reorganizing the Actin Cytoskeleton. Biol. Pharm. Bull. 39 (6), 1029-1034. doi:10.1248/bpb.b16-00047

Asano, T., Kanemitsu, Y., Takemura, M., Yokota, M., Fukumitsu, K., Takeda, N., et al. (2017). Serum Periostin as a Biomarker for Comorbid Chronic Rhinosinusitis in Patients with Asthma. Ann. ATS 14 (5), 667-675. doi:10. 1513/annalsats.201609-720oc

Beermann, J., Piccoli, M.-T., Viereck, J., and Thum, T. (2016). Non-coding Rnas in Development and Disease: Background, Mechanisms, and Therapeutic Approaches. Physiol. Rev. 96 (4), 1297-1325. doi:10.1152/ physrev.00041.2015

Cao, P.-P., Li, H.-B., Wang, B.-F., Wang, S.-B., You, X.-J., Cui, Y.-H., et al. (2009). Distinct Immunopathologic Characteristics of Various Types of Chronic Rhinosinusitis in Adult Chinese. J. Allergy Clin. Immunol. 124 (3), 478-484. doi:10.1016/j.jaci.2009.05.017

Chen, C., Yin, P., Hu, S., Sun, X., and Li, B. (2020). Circular RNA-9119 Protects IL$1 \beta$-treated Chondrocytes from Apoptosis in an Osteoarthritis Cell Model by Intercepting the microRNA-26a/PTEN axis. Life Sci. 256, 117924. doi:10.1016/j. lfs. 2020.117924

Chen, F., Hong, H., Sun, Y., Hu, X., Zhang, J., Xu, G., et al. (2017). Nasal Interleukin 25 as a Novel Biomarker for Patients with Chronic Rhinosinusitis with Nasal Polyps and Airway Hypersensitiveness. Ann. Allergy Asthma Immunol. 119 (4), 310-316. doi:10.1016/j.anai.2017.07.012

Cho, J.-S., Kim, J.-A., Park, J.-H., Park, I.-H., Han, I.-H., and Lee, H.-M. (2016). Tolllike Receptor 4-mediated Expression of Interleukin-32 via the C-Jun N-Terminal Kinase/protein Kinase B/cyclic Adenosine Monophosphate Response Element Binding Protein Pathway in Chronic Rhinosinusitis with Nasal Polyps. Int. Forum Allergy Rhinol. 6 (10), 1020-1028. doi:10.1002/alr.21792

Cho, S.-W., Kim, D. W., Kim, J.-W., Lee, C. H., and Rhee, C.-S. (2017). Classification of Chronic Rhinosinusitis According to a Nasal Polyp and Tissue Eosinophilia: Limitation of Current Classification System for Asian Population. Asia Pac. Allergy 7 (3), 121-130. doi:10.5415/apallergy.2017.7.3.121

Cho, S. H., Kim, D. W., Lee, S. H., Kolliputi, N., Hong, S. J., Suh, L., et al. (2015). Age-related Increased Prevalence of Asthma and Nasal Polyps in Chronic Rhinosinusitis and its Association with Altered IL-6 Trans-signaling. Am. J. Respir. Cel Mol Biol 53 (5), 601-606. doi:10.1165/rcmb.2015-0207rc

Dennis, S. K., Lam, K., and Luong, A. (2016). A Review of Classification Schemes for Chronic Rhinosinusitis with Nasal Polyposis Endotypes. Laryngoscope Invest. Otolaryngol. 1 (5), 130-134. doi:10.1002/lio2.32

Deyev, I. E., Popova, N. V., Serova, O. V., Zhenilo, S. V., Regoli, M., Bertelli, E., et al. (2017). Alkaline $\mathrm{pH}$ Induces IRR-Mediated Phosphorylation of IRS-1 and Actin Cytoskeleton Remodeling in a Pancreatic Beta Cell Line. Biochimie 138, 62-69. doi:10.1016/j.biochi.2017.04.002

Drake, V. E., Rafaels, N., and Kim, J. (2016). Peripheral Blood Eosinophilia Correlates with Hyperplastic Nasal Polyp Growth. Int. Forum Allergy Rhinol. 6 (9), 926-934. doi:10.1002/alr.21793

Elion, R. A., Althoff, K. N., Zhang, J., Moore, R. D., Gange, S. J., Kitahata, M. M., et al. (2018). Recent Abacavir Use Increases Risk of Type 1 and Type 2 Myocardial Infarctions Among Adults with HIV. Jaids J. Acquired Immune Deficiency Syndromes 78 (1), 62-72. doi:10.1097/qai.0000000000001642

Ergun, S., and Oztuzcu, S. (2015). Oncocers: Cerna-Mediated Cross-Talk by Sponging Mirnas in Oncogenic Pathways. Tumor Biol. 36 (5), 3129-3136. doi:10.1007/s13277-015-3346-x

\section{SUPPLEMENTARY MATERIAL}

The Supplementary Material for this article can be found online at: https://www.frontiersin.org/articles/10.3389/fmolb.2021.643504/ full\#supplementary-material

Ferreira, A. F., Calin, G. A., Picanço-Castro, V., Kashima, S., Covas, D. T., and de Castro, F. A. (2018). Hematopoietic Stem Cells from Induced Pluripotent Stem Cells - Considering the Role of Microrna as a Cell Differentiation Regulator. J. Cel Sci 131 (4), jcs203018. doi:10.1242/jcs.203018

Fokkens, W. J., Lund, V. J., Mullol, J., Bachert, C., Alobid, I., Baroody, F., et al. (2012). EPOS 2012: European Position Paper on Rhinosinusitis and Nasal Polyps 2012. A Summary for Otorhinolaryngologists. 2012. A Summary for Otorhinolaryngologists. Rhinology 50 (1), 1-12. doi:10.4193/rhino50e2

He, Q., Liu, N., Hu, F., Shi, Q., Pi, X., Chen, H., et al. (2021). Circ_0061012 Contributes to IL-22-induced Proliferation, Migration and Invasion in Keratinocytes through miR-194-5p/GAB1 axis in Psoriasis. Biosci. Rep. 41 (1), BSR20203130. doi:10.1042/bsr20203130

Jeck, W. R., Sorrentino, J. A., Wang, K., Slevin, M. K., Burd, C. E., Liu, J., et al. (2013). Circular Rnas Are Abundant, Conserved, and Associated with Alu Repeats. RNA 19 (2), 141-157. doi:10.1261/rna.035667.112

Jiao, J., Wang, M., Duan, S., Meng, Y., Meng, N., Li, Y., et al. (2018). Transforming Growth Factor-B1 Decreases Epithelial Tight junction Integrity in Chronic Rhinosinusitis with Nasal Polyps. J. Allergy Clin. Immunol. 141 (3), 1160-1163. doi:10.1016/j.jaci.2017.08.045

Kato, A., Peters, A., Suh, L., Carter, R., Harris, K. E., Chandra, R., et al. (2008). Evidence of a Role for B Cell-Activating Factor of the TNF Family in the Pathogenesis of Chronic Rhinosinusitis with Nasal Polyps. J. Allergy Clin. Immunol. 121 (6), 1385-1392. doi:10.1016/j.jaci.2008.03.002

Kim, B., Lee, H.-J., Im, N.-R., Lee, D. Y., Kang, C. Y., Park, I.-H., et al. (2018). Effect of Matrix Metalloproteinase Inhibitor on Disrupted E-Cadherin after Acid Exposure in the Human Nasal Epithelium. The Laryngoscope 128 (1), E1-E7. doi:10.1002/lary.26932

Korde, A., Ahangari, F., Haslip, M., Zhang, X., Liu, Q., Cohn, L., et al. (2020). An Endothelial microRNA-1-Regulated Network Controls Eosinophil Trafficking in Asthma and Chronic Rhinosinusitis. J. Allergy Clin. Immunol. 145 (2), 550-562. doi:10.1016/j.jaci.2019.10.031

Kuhar, H. N., Tajudeen, B. A., Mahdavinia, M., Gattuso, P., Ghai, R., and Batra, P. S. (2017). Inflammatory Infiltrate and Mucosal Remodeling in Chronic Rhinosinusitis with and without Polyps: Structured Histopathologic Analysis. Int. Forum Allergy Rhinol. 7 (7), 679-689. doi:10.1002/alr.21943

Kulcheski, F. R., Christoff, A. P., and Margis, R. (2016). Circular Rnas Are Mirna Sponges and Can Be Used as a New Class of Biomarker. J. Biotechnol. 238 (20), 42-51. doi:10.1016/j.jbiotec.2016.09.011

Lechuga, S., and Ivanov, A. I. (2017). Disruption of the Epithelial Barrier during Intestinal Inflammation: Quest for New Molecules and Mechanisms. Biochim. Biophys. Acta (Bba) - Mol. Cel Res. 1864 (7), 1183-1194. doi:10.1016/j.bbamcr. 2017.03.007

Li, G., Qin, Y., Qin, S., Zhou, X., Zhao, W., and Zhang, D. (2020). Circ_WBSCR17 Aggravates Inflammatory Responses and Fibrosis by Targeting miR-185-5p/ SOX6 Regulatory axis in High Glucose-Induced Human Kidney Tubular Cells. Life Sci. 259, 118269. doi:10.1016/j.lfs.2020.118269

Li, W., Chang, N., Tian, L., Yang, J., Ji, X., Xie, J., et al. (2017). miR-27b-3p, miR181a-1-3p, and miR-326-5p Are Involved in the Inhibition of Macrophage Activation in Chronic Liver Injury. J. Mol. Med. 95 (10), 1091-1105. doi:10. 1007/s00109-017-1570-0

Liu, B., Ye, B., Zhu, X., Yang, L., Li, H., Liu, N., et al. (2020). An Inducible Circular RNA circKcnt2 Inhibits ILC3 Activation to Facilitate Colitis Resolution. Nat. Commun. 11 (1), 4076. doi:10.1038/s41467-020-17944-5

Liu, J.-H., Cao, L., Zhang, C.-H., Li, C., Zhang, Z.-H., and Wu, Q. (2020). Dihydroquercetin Attenuates Lipopolysaccharide-Induced Acute Lung Injury through Modulating FOXO3-Mediated NF-Kb Signaling via miR132-3p. Pulm. Pharmacol. Ther. 64, 101934. doi:10.1016/j.pupt.2020.101934

Lou, H., Meng, Y., Piao, Y., Wang, C., Zhang, L., and Bachert, C. (2015). Predictive Significance of Tissue Eosinophilia for Nasal Polyp Recurrence in the Chinese Population. Am. J. Rhinol Allergy 29 (5), 350-356. doi:10.2500/ajra.2015.29.4231 
Lou, H., Zhang, N., Bachert, C., and Zhang, L. (2018). Highlights of Eosinophilic Chronic Rhinosinusitis with Nasal Polyps in Definition, Prognosis, and Advancement. Int. Forum Allergy Rhinol. 8 (11), 1218-1225. doi:10.1002/alr. 22214

Martínez-García, C., Izquierdo-Lahuerta, A., Vivas, Y., Velasco, I., Yeo, T. K., and Chen, S. (2015). Renal Lipotoxicity-Associated Inflammation and Insulin Resistance Affects Actin Cytoskeleton Organization in Podocytes. PLoS One 10 (11), e0142291. doi:10.1371/journal.pone.0142291

Martínez-Rivera, V., Negrete-García, M., Ávila-Moreno, F., and Ortiz-Quintero, B. (2018). Secreted and Tissue Mirnas as Diagnosis Biomarkers of Malignant Pleural Mesothelioma. Int. J. Mol. Sci. 19 (2), 595-622. doi:10.3390/ ijms19020595

Milonski, J., Zielinska-Blizniewska, H., Majsterek, I., Przybyłowska-Sygut, K., Sitarek, P., Korzycka-Zaborowska, B., et al. (2015). Expression of POSTN, IL-4, and IL-13 in Chronic Rhinosinusitis with Nasal Polyps. DNA Cel Biol. 34 (5), 342-349. doi:10.1089/dna.2014.2712

Oude Voshaar, M. A. H., Das Gupta, Z., Bijlsma, J. W. J., Boonen, A., Chau, J., Courvoisier, D. S., et al. (2019). International Consortium for Health Outcome Measurement Set of Outcomes that Matter to People Living with Inflammatory Arthritis: Consensus from an International Working Group. Arthritis Care Res. 71 (12), 1556-1565. doi:10.1002/acr.23799

Oyer, S. L., Nagel, W., and Mulligan, J. K. (2013). Differential Expression of Adhesion Molecules by Sinonasal Fibroblasts Among Control and Chronic Rhinosinusitis Patients. Am. J. Rhinol Allergy 27 (5), 381-386. doi:10.2500/ajra. 2013.27.3934

Peng, K., Jiang, P., Du, Y., Zeng, D., Zhao, J., Li, M., et al. (2021). Oxidized Lowdensity Lipoprotein Accelerates the Injury of Endothelial Cells via circ-USP36/ miR -98-5p/VCAM1 axis. IUBMB Life 73 (1), 177-187. doi:10.1002/iub.2419

Plager, D. A., Kahl, J. C., Asmann, Y. W., Nilson, A. E., Pallanch, J. F., Friedman, O., et al. (2010). Gene Transcription Changes in Asthmatic Chronic Rhinosinusitis with Nasal Polyps and Comparison to Those in Atopic Dermatitis. PLoS One 5 (7), e11450. doi:10.1371/journal.pone.0011450

Schleimer, R. P. (2017). Immunopathogenesis of Chronic Rhinosinusitis and Nasal Polyposis. Annu. Rev. Pathol. Mech. Dis. 12, 331-357. doi:10.1146/annurevpathol-052016-100401

Schnoor, M. (2015). Endothelial Actin-Binding Proteins and Actin Dynamics in Leukocyte Transendothelial Migration. J. Immunol. 194 (8), 3535-3541. doi:10. 4049/jimmunol.1403250

Shah, S. A., Ishinaga, H., and Takeuchi, K. (2016). Pathogenesis of Eosinophilic Chronic Rhinosinusitis. J. Inflamm. (Lond) 13 (1), 11. doi:10.1186/s12950-0160121-8

Shi, L.-L., Xiong, P., Zhang, L., Cao, P.-P., Liao, B., Lu, X., et al. (2013). Features of Airway Remodeling in Different Types of Chinese Chronic Rhinosinusitis Are Associated with Inflammation Patterns. Allergy 68 (1), 101-109. doi:10.1111/all.12064

Shimizu, S., Kouzaki, H., Kato, T., Tojima, I., and Shimizu, T. (2016). HMGB1TLR4 Signaling Contributes to the Secretion of Interleukin 6 and Interleukin 8 by Nasal Epithelial Cells. Am. J. Rhinol Allergy 30 (3), 167-172. doi:10.2500/ ajra.2016.30.4300

Sorrenson, B., Cognard, E., Lee, K. L., Dissanayake, W. C., Fu, Y., Han, W., et al. (2016). A Critical Role for $\beta$-Catenin in Modulating Levels of Insulin Secretion from $\beta$-Cells by Regulating Actin Cytoskeleton and Insulin Vesicle Localization. J. Biol. Chem. 291 (50), 25888-25900. doi:10.1074/jbc.m116.758516

Suzuki, H., Koizumi, H., Ikezaki, S., Tabata, T., Ohkubo, J.-i., Kitamura, T., et al. (2016). Electrical Impedance and Expression of Tight junction Components of the Nasal Turbinate and Polyp. ORL J. Otorhinolaryngol. Relat. Spec. 78 (1), 16-25. doi:10.1159/000442024

Tian, T., Zi, X., Peng, Y., Wang, Z., Hong, H., Yan, Y., et al. (2018). H3N2 Influenza Virus Infection Enhances Oncostatin M Expression in Human Nasal Epithelium. Exp. Cel Res. 371 (2), 322-329. doi:10.1016/j.yexcr.2018.08.022
Tsybikov, N. N., Egorova, E. V., Kuznik, B. I., Fefelova, E. V., and Magen, E. (2016) Neuron-specific Enolase in Nasal Secretions as a Novel Biomarker of Olfactory Dysfunction in Chronic Rhinosinusitis. Am. J. Rhinol Allergy 30 (1), 65-69. doi:10.2500/ajra.2016.30.4264

Wu, G., Sun, Y., Xiang, Z., Wang, K., Liu, B., Xiao, G., et al. (2019). Preclinical Study Using Circular Rna 17 and Micro Rna 181c-5p to Suppress the EnzalutamideResistant Prostate Cancer Progression. Cell Death Dis 10 (2), 37. doi:10.1038/ s41419-018-1048-1

Wu, R., Zhang, F., Cai, Y., Long, Z., Duan, Z., Wu, D., et al. (2021). Circ_0134111 Knockdown Relieves IL-1 $\beta$-induced Apoptosis, Inflammation and Extracellular Matrix Degradation in Human Chondrocytes through the Circ_0134111-miR515-5p-SOCS1 Network. Int. Immunopharmacology 95, 107495. doi:10.1016/j. intimp.2021.107495

Xia, S., Feng, J., Lei, L., Hu, J., Xia, L., Wang, J., et al. (2017). Comprehensive Characterization of Tissue-specific Circular Rnas in the Human and Mouse Genomes. Brief Bioinform 18 (6), 984-992. doi:10.1093/bib/bbw081

Xu, M., Chen, D., Zhou, H., Zhang, W., Xu, J., and Chen, L. (2017). The Role of Periostin in the Occurrence and Progression of Eosinophilic Chronic Sinusitis with Nasal Polyps. Sci. Rep. 7 (1), 9479. doi:10.1038/s41598-017-08375-2

Yan, D., Ye, Y., Zhang, J., Zhao, J., Yu, J., and Luo, Q. (2020). Human Neutrophil Elastase Induces MUC5AC Overexpression in Chronic Rhinosinusitis through miR-146a. Am. J. Rhinol Allergy 34 (1), 59-69. doi: $10.1177 / 1945892419871798$

Yan, J. J., Qiao, M., Li, R. H., Zhao, X. T., Wang, X. Y., and Sun, Q. (2019). Downregulation of miR-145-5p Contributes to Hyperproliferation of Keratinocytes and Skin Inflammation in Psoriasis. Br. J. Dermatol. 180 (2), 365-372. doi:10.1111/bjd.17256

Yang, J., Cheng, M., Gu, B., Wang, J., Yan, S., and Xu, D. (2020). CircRNA_09505 Aggravates Inflammation and Joint Damage in Collagen-Induced Arthritis Mice via miR-6089/AKT1/NF-Kb axis. Cel Death Dis 11 (10), 833. doi:10.1038/ s41419-020-03038-z

Yang, R., Xing, L., Zheng, X., Sun, Y., Wang, X., and Chen, J. (2019). The circRNA circAGFG1 Acts as a Sponge of miR-195-5p to Promote Triple-Negative Breast Cancer Progression through Regulating CCNE1 Expression. Mol. Cancer 18 (1), 4. doi:10.1186/s12943-018-0933-7

Yao, Y., Xie, S., and Wang, F. (2019). Identification of Key Genes and Pathways in Chronic Rhinosinusitis with Nasal Polyps Using Bioinformatics Analysis. Am. J. Otolaryngol. 40 (2), 191-196. doi:10. 1016/j.amjoto.2018.12.002

Zhang, X.-H., Zhang, Y.-N., Li, H.-B., Hu, C.-Y., Wang, N., Cao, P.-P., et al. (2012). Overexpression of Mir-125b, a Novel Regulator of Innate Immunity, in Eosinophilic Chronic Rhinosinusitis with Nasal Polyps. Am. J. Respir. Crit. Care Med. 185 (2), 140-151. doi:10.1164/rccm. 201103-0456oc

Zhang, Y.-N., Cao, P.-P., Zhang, X.-H., Lu, X., and Liu, Z. (2012). Expression of microRNA Machinery Proteins in Different Types of Chronic Rhinosinusitis. The Laryngoscope 122 (12), 2621-2627. doi:10.1002/lary.23517

Conflict of Interest: The authors declare that the research was conducted in the absence of any commercial or financial relationships that could be construed as a potential conflict of interest.

Copyright (c) $2021 \mathrm{Yu}$, Kang, Xiong, Luo, Dai and Ye. This is an open-access article distributed under the terms of the Creative Commons Attribution License (CC BY). The use, distribution or reproduction in other forums is permitted, provided the original author(s) and the copyright owner(s) are credited and that the original publication in this journal is cited, in accordance with accepted academic practice. No use, distribution or reproduction is permitted which does not comply with these terms. 